\title{
Effectiveness of Yi-Zhi-An-Shen granules on cognition and sleep quality in older adults with amnestic mild cognitive impairment: protocol for a randomized, double-blind, placebo-controlled trial
}

Shengnan Yue ${ }^{1,2}$, Ting $\mathrm{He}^{1}$, Baiyang $\mathrm{Li}^{1}$, Yanqin $\mathrm{Qu}^{1}$, Hongmei Peng ${ }^{1}$, Jinxin Chen ${ }^{1}$, Ming Lei ${ }^{2}$, Chongli Chen ${ }^{2}$ and Wenbin $\mathrm{Wu}^{1,2^{*}}$

\begin{abstract}
Background: Amnestic mild cognitive impairment (aMCl) is a syndrome characterized by significant forgetfulness that does not meet the criteria of dementia. Individuals with $\mathrm{aMCl}$ are at greater risk of progressing to dementia. Current studies suggest that good sleep quality is linked with preserved cognition in the elderly, and sleep complaints are common among the elderly with amnesia. Therefore, improving their sleep may be helpful for maintaining and improving their cognitive capacity. According to the theory of traditional Chinese medicine, Yi-Zhi-An-Shen is an herbal compound which may ameliorate forgetfulness and sleep disorders. As growing evidence indicates that the gut microbiome is associated with major mental symptoms, a hypothesis was proposed that Yi-ZhiAn-Shen granules (YZASG) might work by alternating microbial abundance and diversity. In this study, the investigators intend to assess the efficacy of YZASG on global cognition in the elderly suffering from aMCl and evaluate its safety as well as its potential mechanisms via sleep quality, fecal microbial 165 ribosomal DNA and metagenomics analyses, and serum markers.
\end{abstract}

Methods/design: This study is a randomized, double-blind, placebo-controlled clinical trial. A total of 80 patients (aged 60-85 years) will be recruited and allocated randomly to a treatment group and a placebo group in a 1:1 ratio and will then be administered YZASG or isodose placebo three times a day. The intervention course is 16 weeks, with an 18 months follow-up. The primary outcome is the Alzheimer's Disease Assessment Scale-Cognitive Subscale. Secondary outcome measures are the Mini-Mental State Examination, Montreal Cognitive Assessment, Pittsburgh Sleep Quality Index, serum concentrations of immunological factors and inflammatory cytokines, and fecal microbiota. Fecal microbiota will only be collected at the baseline and endpoint of the intervention.

Discussion: The results of this trial will be conducive to assessing the safety and effectiveness on cognition of YZASG in intervening aMCl among the elderly and determining if it takes effect via the improvement of sleep quality, regulation of gut microbiota, and concentration of certain serum markers.

Trial registration: ClinicalTrials.gov, NCT03601000. Registered on 26 July 2018.

Keywords: Amnestic mild cognitive impairment, Sleep, Gut microbiome, Older adults

\footnotetext{
* Correspondence: wwb1201@vip.sina.com

${ }^{1}$ School of Clinical Medicine, Chengdu University of Traditional Chinese

Medicine, No. 1177 Liu-tai Avenue, Chengdu 611137, Sichuan Province,

People's Republic of China

${ }^{2}$ Department of Geriatrics, Hospital of Chengdu University of Traditional

Chinese Medicine, No. 39 Shi-er-qiao Road, Chengdu 610072, Sichuan

Province, People's Republic of China
}

(c) The Author(s). 2019 Open Access This article is distributed under the terms of the Creative Commons Attribution 4.0 International License (http://creativecommons.org/licenses/by/4.0/), which permits unrestricted use, distribution, and

reproduction in any medium, provided you give appropriate credit to the original author(s) and the source, provide a link to the Creative Commons license, and indicate if changes were made. The Creative Commons Public Domain Dedication waiver (http://creativecommons.org/publicdomain/zero/1.0/) applies to the data made available in this article, unless otherwise stated. 


\section{Background}

Alzheimer's disease (AD), one common type of dementia worldwide, is a neurodegenerative disease characterized by insidious onset and progressive cognitive decline [1]. It not only detrimentally affects physical condition and quality of life in patients, but it also places a huge burden on both the family and society [2]. The prevalence of $\mathrm{AD}$ is $3.21 \%$ reportedly among the old in China [3]. It was proposed recently that research strategies for $A D$ should focus on the preclinical stages - preclinical AD and mild cognitive impairment (MCI) due to $\mathrm{AD}-$ which are relatively ideal for intervention [4].

Patients with MCI experience cognitive decline which is more severe than expected for an individual's age and education level but which does not obviously affect daily function [5]. Also, the elderly with MCI are at a high risk of developing dementia [6]. Nearly $90 \%$ of older adults with amnestic MCI (aMCI), i.e., MCI with memory complaints and the main subtype of $\mathrm{MCI}$, reportedly progress to $\mathrm{AD}$ and share similar pathophysiological characteristics with AD patients [7]. Nowadays, standard clinical management of MCI includes managing its risk factors, as there are still only limited pharmaceutical options for treating $\mathrm{MCI}$, according to the National Institute on Aging-Alzheimer's Association (NIA-AA) working group guidelines [8]. Thus, effective drugs and other interventions are expected to be discovered to reduce the rate of progression from MCI to dementia.

Brain areas and systems of neurotransmitters involving regulation of the sleep-wake cycle relate primarily to memory and cognition [9-11]. Individuals with various types of cognitive impairment generally have sleep disturbance $[9,12-14]$, and one study also suggested that the severity of these sleep disorders closely relates to that of cognitive decline [14]. Moreover, older adults with bad sleep quality always suffer poor cognitive performance $[15,16]$, which might be related to higher levels of $\mathrm{AD}$-associated amyloid- $\beta$ in the brain [17]. Structural imaging data indicated that insomnia is also associated with a decreased volume of brain tissue, including the hippocampus [18]. A disorder of the circadian rhythm often accompanies a high risk of cognitive decline and negatively affects cognition in multiple ways. Therefore, a sleeping problem is one risk factor of impaired cognitive function.

Several studies suggested that besides improving the cognitive performance and ability of daily living in individuals with $\mathrm{AD}$, acetylcholinesterase inhibitors (ChEIs), like donepezil, galantamine, and rivastigmine, also improved the mental behavioral symptoms and sleep quality of these patients [19-21]. Furthermore, some trials indicated that melatonin could improve cognitive symptoms in patients with $\mathrm{MCI}$ and might delay their conversion to dementia [22, 23]. As sleep disturbance appears to be linked with both aging and cognitive decline, strengthening the sleep quality of the elderly might be an effective therapeutic target to slow the deterioration or improve cognitive impairment.

According to recent studies on $\mathrm{MCI}$ or $\mathrm{AD}$ which involved donepezil [7], galantamine [24], memantine [25], and solanezumab [26], pharmaceutical interventions showed poor amelioration of cognitive deficits. It might be that the single pharmacological target of these drugs did not adequately address the specific multiple pathophysiological characteristics. However, traditional Chinese medicine (TCM) is known for its multitargeted approach and could be used to address the complicated pathophysiological changes.

In the light of TCM theory, kidney deficiency is the basis of amnesia and other cognitive deficits, while phlegm and blood stasis are significant pathological factors. For this reason, experiments and trials have been carried out and have shown specific effects against cognitive decline, involving, e.g., Alpiniae oxyphyllae fructus [27], Ligusticum wallichii [28-30], Curcuma [31, 32], Fructus gardeniae [33, 34], and Radix notoginseng [35, 36]. Meanwhile, an herbal formula named Yi-Zhi-An-Shen granules (YZASG) has been developed, composed of Lophatherum gracile and the medical herbs mentioned above, which is designed for aiding the treatment of cognitive deficits. Table 1 details the pharmacological targets of the components in YZASG, which could be involved in the management of MCI. There have been preclinical research studies contributing to the standardization of YZASG [37] and the mechanisms of its potential actions in animal experiments [38]. Some indications have also manifested its safety and efficacy for improving cognitive function in clinical practice without causing daytime dysfunction.

Based on the theory of TCM, the actions of YZASG include soothing the nerves, that is, making a person calm down and helping him/her sleep. Besides, according to the emerging hypothesis of the brain-gut axis, numerous studies have shown that changes of the gut microbiome and its metabolites exert an influence on cognition impairment [39-41]. Because it is administered orally, we note that the mechanism of action of YZASG might be via the gut microbiota. Consequently, a clinical trial with rigorous design is needed to confirm its safety in old individuals with MCI and its efficacy on cognitive performance, and also to explore its potential mechanisms.

\section{Methods \\ Objectives}

The primary objective of this study is to evaluate the efficacy of YZASG in optimizing cognitive performance over time in elderly individuals with aMCI. Secondly, the 
Table 1 Multitarget mechanisms underlying pharmacological effects of YZASG components

\begin{tabular}{ccccc}
\hline $\begin{array}{c}\text { Pharmacological effects relevant } \\
\text { to MCI }\end{array}$ & $\begin{array}{c}\text { Alpiniae oxyphyllae } \\
\text { fructus }\end{array}$ & $\begin{array}{c}\text { Ligusticum } \\
\text { wallichii }\end{array}$ & $\begin{array}{c}\text { Curcuma } \\
\text { Fructus gardenia }\end{array}$ & $\begin{array}{c}\text { Radix } \\
\text { notoginseng }\end{array}$ \\
\hline Anti-inflammatory & $\sqrt{ }$ & $\sqrt{ }$ & $\sqrt{ }$ & $\sqrt{ }$ \\
Anti-oxidant & $\sqrt{ }$ & $\sqrt{ }$ & $\sqrt{ }$ & $\sqrt{ }$ \\
Anti-apoptotic & $\sqrt{ }$ & & & $\sqrt{ }$ \\
Improving \\
cholinergic transmission
\end{tabular}

investigators intend to assess whether YZASG can improve sleep quality among aMCI patients, and this herbal formula's safety will also be assessed. Finally, participants' serum samples and fecal genomic DNA will be extracted to analyze the differences of the indices of metabolism, cellular immune function, and gut microbiota between old individuals with aMCI and those with normal cognition.

\section{Design}

This study is a randomized, double-blind, placebocontrolled trial with a 16-week intervention and an 18 months follow-up assessment. The current protocol (version v1.1) meets the principles of the Declaration of Helsinki, is in accordance with Standard Protocol Items: Recommendations for Interventional Trials (SPIRIT) guidelines (see Additional file 1), and was approved by the Medical Ethics Committee of Hospital of Chengdu University of TCM. Signed informed consent forms will be obtained from participants, and during the consent process, the caregiver or informant of the potential participant should be present. The participants will be recruited from the communities, outpatient clinics of Hospital of Chengdu University of TCM, and the Welfare Institution of Emei Civil in Sichuan Province, China. The Medical Ethics Committee has thoroughly reviewed this study, and the ethical approval covers all these study sites.

The enrolled participants will be randomly assigned into the YZASG group or the placebo group with an allocation ratio of 1:1 using a statistical package. Allocation was concealed using batch numbers generated with SAS 9.2 software (SAS Institute, Cary, NC, USA) by a statistician. A unique code will be assigned to each newly enrolled participant and preserved by the trial management board. The statistician expert who acts as the coder will be shielded from subject recruitment and the statistical analysis, which will be performed by another statistician independent of the study group. Both participants and the research team are blinded to allocation. There will be six measurement sessions during this study, including the intervention period and the 18 months period (Fig. 1). Any changes to the study protocol will be communicated to the study investigative team and the approving ethics committee.

\section{Participants}

The investigators anticipate recruiting 80 participants from Hospital of Chengdu University of TCM, communities, and the Welfare Institution of Emei Civil Administration. All patients will undergo a standard medical examination and neuropsychological testing to ensure the correct diagnosis of aMCI.

The inclusion criteria are:

1. Assigned informed consent from the subject to participate in the study and continued willing consent for participation

2. Age from 60 to 85 years with a diagnosis of aMCI

3. Educational level of at least 6 years 


\begin{tabular}{|c|c|c|c|c|c|c|c|c|}
\hline \multirow{3}{*}{ Time point (weeks) } & \multicolumn{8}{|c|}{ Study Period } \\
\hline & \multirow{2}{*}{$\begin{array}{c}\text { Enrollment } \\
-4\end{array}$} & \multirow{2}{*}{$\begin{array}{c}\text { Baseline } \\
0\end{array}$} & \multicolumn{3}{|c|}{ Intervention Period } & \multicolumn{3}{|c|}{ Follow-up Period } \\
\hline & & & 1 & 8 & 16 & 42 & 68 & 94 \\
\hline \multicolumn{9}{|l|}{ Enrollment: } \\
\hline Eligibility & $x$ & & & & & & & \\
\hline Informed Consent & $\times$ & & & & & & & \\
\hline Allocation & & $x$ & & & & & & \\
\hline \multicolumn{9}{|l|}{$\begin{array}{l}\text { Interventions: } \\
\text { YZASG or placebo }\end{array}$} \\
\hline \multicolumn{9}{|l|}{ Assessments: } \\
\hline \multicolumn{9}{|l|}{ Primary Outcome } \\
\hline ADAS-cog & & $x$ & & & $x$ & $x$ & $x$ & $x$ \\
\hline \multicolumn{9}{|l|}{ Secondary Outcomes } \\
\hline MMSE & & $x$ & & $x$ & $x$ & $x$ & $\times$ & $x$ \\
\hline MoCA & & $x$ & & $x$ & $x$ & $x$ & $x$ & $x$ \\
\hline CDR & & $x$ & & & $x$ & $x$ & $x$ & $x$ \\
\hline PSQI & & $x$ & & $x$ & $x$ & $x$ & $\times$ & $x$ \\
\hline ADL & & $x$ & & $x$ & $x$ & $x$ & $x$ & $x$ \\
\hline Blood Sample & & $x$ & & & $x$ & & & \\
\hline \multicolumn{9}{|l|}{ Metabolic markers } \\
\hline \multicolumn{9}{|l|}{ Inflammatory markers } \\
\hline \multicolumn{9}{|l|}{ Immunologic markers } \\
\hline \multicolumn{9}{|l|}{ Fecal Sample } \\
\hline 16SrDNA & & $x$ & & & $x$ & & & \\
\hline Metagenomics & & $x$ & & & $x$ & & & \\
\hline \multicolumn{9}{|l|}{ Safety Outcomes } \\
\hline Vital signs & & $x$ & $x$ & & $x$ & & & \\
\hline Weight & & $x$ & $x$ & & $x$ & & & \\
\hline Full blood count & & $x$ & $\times$ & & $x$ & & & \\
\hline Coagulation function & & $x$ & $\times$ & & $x$ & & & \\
\hline Liver function test & & $x$ & $\times$ & & $x$ & & & \\
\hline Renal function test & & $x$ & $\times$ & & $x$ & & & \\
\hline ECG & & $x$ & $x$ & & $x$ & & & \\
\hline Brain CT/MRI & & $x$ & & & $x$ & & & \\
\hline
\end{tabular}

Fig. 1 Schedule of interventions and assessments. Abbreviations: ADAS-cog Alzheimer's Disease Assessment Scale-Cognitive Subscale, MMSE MiniMental State Examination, MoCA Montreal Cognitive Assessment, CDR Clinical Dementia Rating, PSQ/ Pittsburgh Sleep Quality Index, ADL activities of daily living, 165 rDNA 165 ribosomal deoxyribonucleic acid, ECG electrocardiography, CT computed tomography, MRI magnetic resonance imaging. Vital signs include an individual's temperature, breaths and pulse per minute, and blood pressure

4. Availability of a caregiver or informant who can assist in completing rating scales for the duration of the study

5. Cognitive complaints reported by the subject and confirmed by the caregiver or informant

6. Clinical Dementia Rating (CDR) global score of 0.5 and memory item score of 0.5

7. Mini-Mental State Examination (MMSE) score of 24-30 (for participants with educational level of 6 years, MMSE score of 20-30)
8. Diagnostic and Statistical Manual of Mental Disorders, Version 5 (DSM-V) criteria of dementia not fulfilled.

A patient will be excluded if he or she:

1. Has been previously enrolled in this study and received the investigational product

2. Has received an investigational product within 30 days prior to screening 


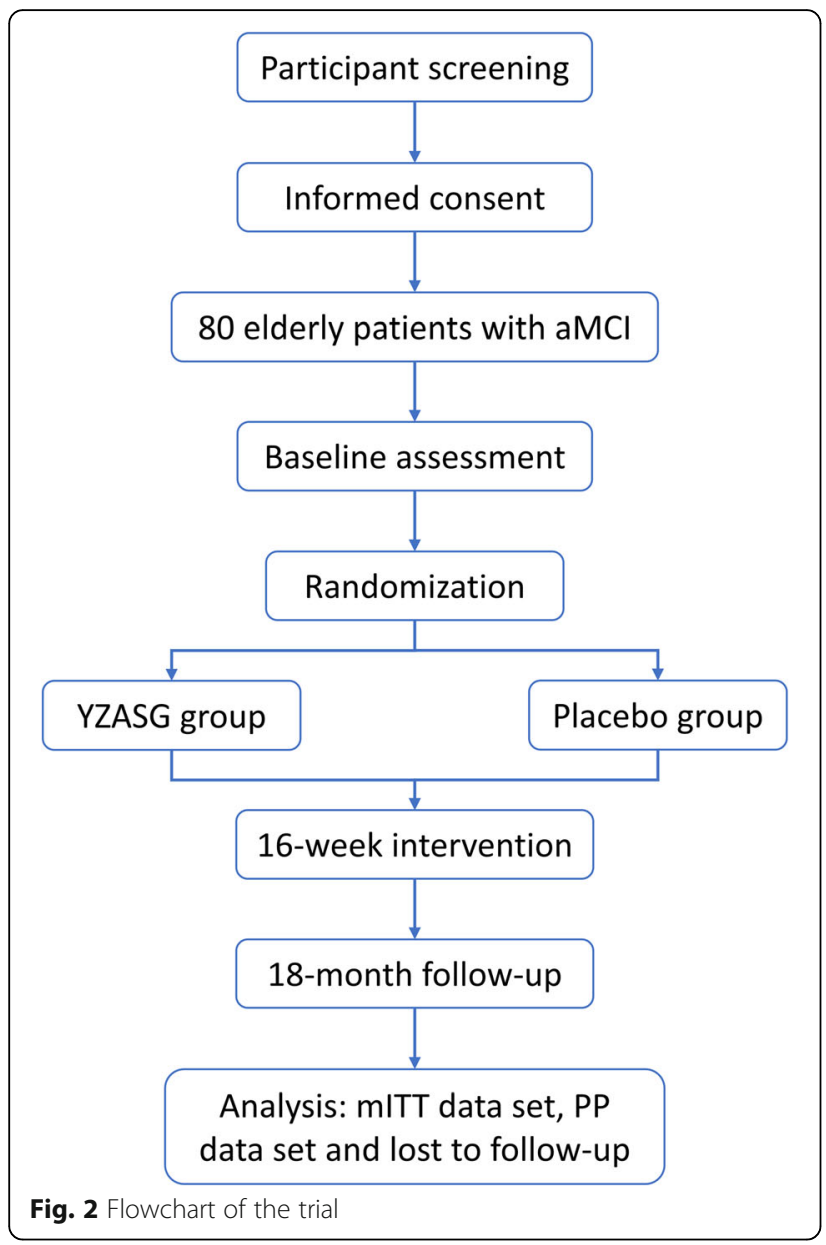

3. Has received disease-modifying therapy in the past 6 months (e.g., donepezil, rivastigmine, galantamine, memantine, or any other existing drugs that are declared to have the function of improving cognition)

4. Has a known allergy to the study drug or any of its constituents

5. Has a history of alcohol abuse or alcohol dependency in the 3 years prior to study entry, or is an alcoholic or drug addict, as determined by the investigator

6. Has ongoing clinically significant (as judged by the investigator), metabolic, or any other disease that could currently cause impaired memory (e.g., untreated thyroid disease, vitamin or other nutritional deficiencies, chronic kidney or liver disease)

7. Has memory impairments that can be attributed to a disease or condition other than an early-phase neurodegenerative syndrome

8. Has a parkinsonian movement disorder

9. Uses psychoactive medications that would affect the subject's ability to reliably perform neurocognitive testing or create uncertainty in distinguishing between the effects of the psychoactive medication and the subject's underlying cognitive impairment (e.g., benzodiazepines, sedatives, antipsychotics)

10. Has a history of major recurrent depressive disorder (per DSM-V) within the last 5 years prior to screening

11. Has a brain tumor or other intracranial lesion, a disturbance of cerebral spinal fluid circulation (e.g., normal pressure hydrocephalus), and/or a significant history of head trauma or brain surgery

12. Has signs of major cerebrovascular disease, with a modified Hachinski Ischemia Score (mHIS) of more than 4 , or as verified by medical history and/or brain magnetic resonance imaging (MRI) or computed tomography (CT) scan

13. Has severe visual or hearing impairments and cannot cooperate with examinations

14. Has a severe digestive system disease

15. Has received antibiotics within 60 days prior to screening.

Reasons for participant withdrawal and dropout are as follows (1) voluntarily withdrawal, (2) loss of follow-up, (3) poor compliance and presence of severe adverse effects, (4) revealing and uncovering blind in urgency, (5) misdiagnosis, (6) using forbidden drugs or treatments in the course of the trial, (7) taking no medication during the trial, (8) no evaluable records after medication. Reasons for withdrawing participants will be recorded in case report forms (CRFs), and the last of their data will be included in the data analysis. All these criteria will be ascertained by the supervisor of this study.

\section{Interventions}

A flowchart of this trial is presented in Fig. 2. All the participants will receive the same basic treatment, including health education, moderate aerobic exercise (30-60 min per day), and general nutritional support. The participants assigned to the YZASG group will take the Yi-Zhi-An-Shen granule, which is composed of YiZhiRen (Alpinia oxyphylla Miq.) $5 \mathrm{~g}$, SanQi (Panax notoginseng) $3 \mathrm{~g}$, ChuanXiong (Ligusticum chuanxiong Hort.) $10 \mathrm{~g}$, ZhiZi (Gardenia jasminoides Ellis) $10 \mathrm{~g}$, Yulin (Curcuma longa L.) $10 \mathrm{~g}$, and DanZhuYe (Lophatherum gracile) $10 \mathrm{~g}$, while those in the placebo group will take a placebo made from starch which has the same shape, color, smell, taste, texture, package, and lot number as the intervention. Participants will be instructed to dissolve the granules into $100 \mathrm{ml}$ of boiled water and to take the solution orally at a temperature between 30 and $37^{\circ} \mathrm{C}$ three times daily for 16 weeks. Each granule is prepared by $\mathrm{Si}-$ chuan Neo-Green Pharmaceutical Technology Development Co., Ltd., Sichuan, China, according to the standards 
of Good Manufacturing Practice (GMP). During the entire study period, relevant health care and treatment for medical needs will be permitted as long as there are no adverse reactions with YZASG and no hindering of the study process.

The actual dose taken by one patient within the range of 80 to $120 \%$ of the recommended dose is considered eligible for the protocol plan. The package, drug name, function and indication, usage and dosage, storage conditions, validity period, and name of the manufacturer will be marked, and a tag indicating "trial use" will be attached. Drugs must be kept at the appropriate temperature in a dry, cool, and shady place. Drug administrators should take back unused drugs to estimate participant compliance and record this in the CRFs.

\section{Outcomes}

\section{Primary outcome}

Cognitive decline is measured using the Chinese version of the Alzheimer's Disease Assessment Scale-Cognitive Subscale (ADAS-cog11). This scale was also chosen to calculate the sample size, with a 4-point change in score as the measure of clinical significance [42]. The total possible score is 70; a higher score indicates greater severity of impairment. The specific hypothesis is that the increase from baseline to endpoint will be significantly less (by at least 2.5 points) than that for the placebo, which will be considered effective in this study.

The ADAS- $\operatorname{cog} 11$ will be assessed at baseline (before intervention), at 16 weeks (the end of the intervention), and at 6,12 , and 18 months after the intervention.

\section{Secondary outcomes}

Secondary measures include the MMSE, Montreal Cognitive Assessment (MoCA, Changsha version), CDR, Pittsburgh Sleep Quality Index (PSQI), and activities of daily living (ADL) scores, the gut microbiome, and serum markers.

The MMSE is an 11-question measure that tests five areas of cognitive function (orientation, registration, attention and calculation, recall, and language). The maximum score is 30 , and a score below 24 is considered abnormal for dementia screening.

The MoCA (Changsha version) will also be used to evaluate general cognitive function, as it contains visuospatial processing and organizational capability parts and can make up for the shortcomings of the MMSE. The total score for the MoCA is 30, with a higher number indicating a more intact cognitive function. The MoCA has been shown to be a promising tool to detect $\mathrm{MCI}$ and early AD.

The CDR will be used as an assistant evaluation for patients' dementia severity. It scores $0-3$, with higher scores indicating more severity. It is a semi-structured interview performed with the patient and caregiver (informant), characterizing six domains of cognitive and functional performance. The CDR sum of boxes (CDRSB), scored 0-18, will also be applied to assess patients' cognitive status, with higher scores indicating worse functioning.

The PSQI will be used to assess participants' comprehensive quality of sleep, which involves sleep quality, sleep duration, sleep efficiency, sleep disorders, daytime dysfunction, sleeping aids, etc. The total score for the PSQI is 21, with a higher score indicating a worse sleep quality.

ADL will be assessed including basic activities of daily living (BADL) and instrumental activities of daily living (IADL). An individual's BADL will be evaluated mainly by the subjects' performance from the perspectives of bathing, dressing, grooming, initiation, toileting, and feeding, with six items and a sum of scores ranging from 0 (normal) to 24 (complete dependence on others). A modified Lawton IADL scale will be used to measure the IADL of a subject, with eight items and a sum of scores ranging from 0 (normal) to 32 (complete dependence on others).

These clinical tests will be administered by a trained, certified clinician or rater experienced in the assessment of patients with cognitive deficits. The rater who conducts the CDR for a patient cannot complete any other rating scales for the same patient, and will be blinded to the results of all other neuropsychological scales. The previously described scales will be assessed at baseline (before the intervention), at 8 and 16 weeks (during the intervention), and at 6,12, and 18 months after the intervention.

Blood samples will be collected from all participants to further assess the mechanisms of YZASG via changes in serum metabolic, inflammatory, and immunologic markers. All these tests will be entrusted to the laboratory medicine departments of Sichuan Academy of Medical Science \& Sichuan Provincial People's Hospital for conduction.

Fecal genomic DNA will be extracted from frozen stools using a QIAamp DNA Mini Stool Kit (Qiagen, Hiden, Germany), obtained from the patients with aMCI and 15-20 participants with normal cognition at baseline and at the end of the intervention. After polymerase chain reaction (PCR) amplification, DNA fragments will be sequenced on an Illumina HiSeq 2500 instrument and an Illumina HiSeq X instrument for $16 \mathrm{~S}$ ribosomal DNA (16S rDNA) and metagenomics analyses (which will be chosen from some representative samples in the results of the $16 \mathrm{~S} \mathrm{rDNA}$ analysis), respectively, at Biomarker Technologies Co, Ltd. (Beijing, China) to analyze the differences in gut microbiome between patients with aMCI and individuals with normal cognition. When the results are available, the investigators 
will also assess the changes in gut microbiome between the treatment group and the placebo group after the intervention of YZASG in the same way.

\section{Safety outcomes}

To assess the safety of YZASG compared to placebo in subjects with aMCI, the investigators will record the incidence and severity of treatment-emergent adverse events (TEAEs) and clinically important changes in safety assessment results. These safety indicators, including vital signs, weight, clinical laboratory tests, physical and neurological exams, electrocardiography (ECG), and CT/MRI scans, will be gathered at the baseline and at the end of the 16th week.

\section{Statistical considerations and data management Sample size}

Using pre-intervention and post-intervention scores obtained from Miao et al. [43], on the basis of a noninferiority trial principle, one-sided test, at $\alpha=0.05$, at least 33 patients are needed for inclusion in the treatment group who will be administered YZASG to achieve a prospective power of $90 \%$ (i.e., $\beta=0.1$ ) and detect a minimum clinical between-group difference of 1.30 on ADAS-cog [43] at 16 weeks. Allowing for a maximum dropout rate of $20 \%$, the number of subjects in the treatment group has been set to 40 patients with aMCI. With an allocation ratio of 1:1, 80 subjects are required.

\section{Statistical analysis}

The analysis will be conducted by another statistician, from the National Clinical Trial Center of Chinese Medicine (Chengdu, China), who will also be blinded to the whole trial, using SAS 9.2 software (Cary, NC, USA) and SPSS 21.0 software (IBM, Armonk, NY, USA).

The analysis data set will consist of a modified intention-to-treat data set, a per protocol (PP) set, and a safety data set. All mechanism and efficacy analyses will be conducted according to the modified intention-totreat (mITT) principle. The mITT data set will include the participants who have completed at least one observation since the intervention began. The PP population will only include participants who adhered to the trial protocol and completed the clinical trial; the minimum compliance rate for participants taking the investigational drugs in the PP data set is $80 \%$. A safety analysis will be conducted according to the safety data set, which will include any participants who were randomly assigned to and took at least one dose of the investigational drug. Missing values will be replaced by the last observation carried forward (LOCF) method. ADAS-cog (including its monomial item) changes from baseline and the secondary outcomes will be assessed using an analysis of covariance with treatment groups as factors and baseline values as covariates. Mean differences will be used to express effect sizes. The baseline homogeneity of the baseline characteristics and differences between the two groups will be analyzed with Fisher's exact test or the $\chi^{2}$ test for categorical measures and with the $t$ test or Wilcoxon rank-sum test for continuous measures. The statistical significance is defined as a onesided $p$ value of $<0.05$ and $90 \%$ confidence interval.

For the gut microbial $16 \mathrm{~S}$ rDNA analysis, sequenced data will be interpreted using the bioinformatics tools programmed in the Ion Reporter Software. Based on the specified similarity, Quantitative Insights into Microbial Ecology (QIIME) algorithms will be used to classify operational taxonomic units (OTUs) and statistically analyze biological information, then to understand the diversity and abundance of the flora community, and further to determine the bacterial diversity within a sample ( $\alpha$-diversity) and among all the samples ( $\beta$-diversity). The $\alpha$-diversity includes four indicators that represent total number, richness, phylogenetic diversity, and dispersible uniformity of species and community abundance. These four algorithms, including binary Jaccard, Bray-Curtis, weighted UniFrac, and unweighted UniFrac, will be performed to analyze the $\beta$-diversity to compare the similarity of different samples in species diversity. According to the preceding data, principal component analysis will be conducted to observe the differences between floras. Additionally, multivariate data analysis with principal component analysis on the diversity indices and comparisons of genus and species level data will be performed to reveal differences in the microbial composition between individuals with normal cognition and those with aMCI. Metastats software will be used to perform $t$ tests on the species abundance data between the two groups, and a $p$ value will be obtained; then by correcting the $p$ value, a $q$ value will also be obtained. Subsequently, according to the $p$ or $q$ value, species that cause the differences in the microbial composition of the two sets will be screened out. Significant analyses between two groups will be performed at the level of classification of the gate, class, subject, family, genera, and species, respectively. The SparCC algorithm will be used to conduct correlation analyses (including positive and negative correlation) and statistical tests. Next, a coexpression analysis network map will be drawn using Python. The subsequent statistical analyses will be performed with the R Programming Language 3.0.1 (NZL).

For the metagenomics analysis, after getting Clean Reads, a taxonomic analysis will be conducted to measure species composition and abundance information of samples. After the significance test of differences has been performed, a $p$ value will be obtained. Then by correcting the $p$ value, the false discovery rate (FDR) will be obtained. The Benjamini-Hochberg FDR adjustment will 
be used to account for the number of taxa tested in each comparison.

\section{Data management}

All data will be stored on a secure server with two backup copies on external hard drives. Paper-based forms will be digitized and the original copies stored in locked filing cabinets in the archive of Good Clinical Practice (GCP) in the Department of Geriatrics, and managed by a department staff member who is external to the research team. All participants are de-identified upon randomization and referred to on all forms by a participant ID. A password-protected spreadsheet stored on the secure server links all participant names to ID codes so that re-identification can occur if required. As this is a relatively small investigator-initiated trial, a data monitoring committee and auditing process are not required.

\section{Quality control and monitoring}

Each trial center has a project manager who takes charge of the quality of research. All investigators were qualified and trained before the study began. After the baseline measurement, the 16 weeks of intervention medication will be dispensed. Participants will be required to return any unused medication every 2 weeks in order to determine compliance. The number of returned granules will be counted by a department member who is external to the study team. During the whole course of the trial, attentive follow-up will also be conducted every 2 weeks. Participants who exit the study early will be contacted via telephone and requested to complete the exit interview.

To monitor safety, participants will be referred to Hospital of Chengdu University of TCM and the local cooperating hospital of the Welfare Institution of Emei Civil Administration, which is next to the institution. Standard blood safety tests (full blood count, blood coagulation function, liver and renal function tests) and ECG tests will be carried out at the baseline and at the end of the 1st and 16th weeks of intervention, while brain CT/MRI scans will be done at the baseline and at the end of the 16th week. Participants are also encouraged to share their annual physical checkup reports with the investigators in this study.

Adverse events will be closely monitored and recorded throughout the whole course of the study. If a serious adverse event occurs, if the study drug is suspected to be a potential cause and receiving appropriate medical care is identified to be essential for the participant, then unblinding of that participant will occur, and at least two persons should be present. After being treated, that participant will be discontinued from the subsequent investigation and regarded as a dropout case. The investigators will also visit that participant regularly and record his/her reactions and treatments of the adverse events until the endpoint of this study. This task will be performed by another group of physicians who are independent of the groups of investigators who are in charge of enrolment, administering drugs and evaluating participants. A development update safety report must be submitted annually to the Medical Ethics Committee of Hospital of Chengdu University of TCM.

The random code and allocation information will be kept concealed from the study team and participants until the end of this study when all statistical analyses have been finished.

\section{Discussion}

YZAS has shown effectiveness in improving cognitive performance and neuroinflammation in animal experiments. Provided that it can also be proved to address cognitive deficits in this clinical trial, it may be shown to act via multiple mechanisms involved in the pathology of aMCI, which will be determined in the study.

Assuming that old individuals with aMCI almost always experience poor sleep, this study may provide an evidence-based medical approach to improving sleep quality and then maintaining cognition among these patients. However, in terms of testing specifically the differences of fecal microbiome between the old with normal cognition and those with aMCI, this project has a relatively small sample size, which is calculated according to the primary outcome. Owing to the number and the length of the tasks, the participants' burden is a bit high; breaks will be scheduled during each period of follow-up assessments. The results of this randomized controlled trial will inform the development of future interventional studies to prevent or delay cognitive decline among patients with $\mathrm{MCI}$ or dementia.

\section{Trial status}

This trial (protocol version v1.1, 18 December 2017) began recruitment on 21 April 2018 and is currently ongoing. The recruitment is expected to be completed by the end of 2019 .

\section{Additional file}

Additional file 1: SPIRIT 2013 checklist: recommended items to address in a clinical trial protocol and related documents. (DOC $138 \mathrm{~kb}$ )

\section{Acknowledgements}

We sincerely appreciate Dr. Jieping Zeng, who works at the Center of Good Clinical Practice of Hospital of Chengdu University of Traditional Chinese Medicine, for her assistance with the statistical analysis. We also appreciate Mrs. Xitao Ma, a colleague of Dr. Zeng, for her assistance in making the randomization. We truly appreciate Professor Yong Tang for his

recommendations for the study design as well. We feel grateful to all the health care workers involved in this study for their help. We also express special gratitude to all the participants in this study. 


\section{Authors' contributions}

WBW, the lead principal investigator, contributed to the study design and the development of this trial with SNY. SNY drafted the initial protocol, and WBW provided the review. TH and BYL participated in the coordination of the trial and recruited patients with YQQ and JXC. HMP assisted with the collection of data. ML and CLC advised on medical assessment and clinical issues. CLC also worked as the clinical supervisor. All authors reviewed the content and approved the final version of the manuscript.

\section{Funding}

This study is funded by the State Administration of Traditional Chinese Medicine of the People's Republic of China (JDZX2015298), Benefiting the Public Program of Chengdu Science and Technology Bureau (2015-HM01 -00467-SF), Cadres Health Care Research Program of Sichuan Province (2017 -501, 2018-504). Part of the costs concerning the drugs in this study is financially supported by Sichuan Neo-Green Pharmaceutical Technology Development Co., Ltd., Chengdu, China. These funding agencies and the company has had and will have no role in study design, data collection, management, analysis, interpretation of data, or in writing this manuscript.

\section{Availability of data and materials}

The data sets used and/or analyzed during this study are available from the corresponding author on reasonable requests.

\section{Ethics approval and consent to participate}

Ethical approval for this study was provided by Hospital of Chengdu University of Traditional Chinese Medicine Medical Ethics Committee (2017KL-035, 2017KL-036). All participants for this study will provide written informed consent.

\section{Consent for publication}

Not applicable.

\section{Competing interests}

The authors declare that they have no competing interests.

Received: 11 December 2018 Accepted: 20 July 2019

Published online: 20 August 2019

\section{References}

1. Querfurth HW, LaFerla FM. Alzheimer's disease. N Engl J Med. 2010; 362:329-44

2. Alzheimer's Disease International. World Alzheimer's Report 2016. London: Alzheimer's Disease International; 2017.

3. Jia J, Wang F, Wei C, et al. The prevalence of dementia in urban and rural areas of China. Alzheimers Dement. 2014;10:1-9.

4. Silverberg N, Elliott C, Ryan L, Masliah E, Hodes R. NIA commentary on the NIA-AA Research Framework: towards a biological definition of Alzheimer's disease. Alzheimers Dement. 2018;14:576-8.

5. Magaki S, Mueller C, Dickson C, Kirsch W. Increased production of inflammatory cytokines in mild cognitive impairment. Exp Gerontol. 2007;42:233-40.

6. Petersen RC. Mild cognitive impairment: transition from aging to Alzheimer's disease. In: labal K, Sisodia SS, Winblad B, editors. Alzheimer's disease: advances in etiology, pathogenesis and therapeutics. Hoboken: Wiley; 2001. p. 141-51.

7. Petersen RC, Thomas RG, Grundman M, et al. Vitamin E and donepezil for the treatment of mild cognitive impairment. N Engl J Med. 2005: 352:2379-88.

8. Petersen RC, Lopez O, Armstrong MJ, et al. Practice guideline update summary: mild cognitive impairment: Report of the Guideline Development, Dissemination, and Implementation Subcommittee of the American Academy of Neurology. Neurology. 2018;90:126-35.

9. Zhong G, Naismith SL, Rogers NL, Lewis SJ. Sleep-wake disturbances in common neurodegenerative diseases: a closer look at selected aspects of the neural circuitry. J Neurol Sci. 2011;307:9-14.

10. Lim J, Dinges DF. A meta-analysis of the impact of short-term sleep deprivation on cognitive variables. Psychol Bull. 2010;136:375-89.

11. Diekelmann S, Born J. The memory function of sleep. Nat Rev Neurosci. 2010;11:114-26.

12. Beaulieu-Bonneau S, Hudon C. Sleep disturbances in older adults with mild cognitive impairment. Int Psychogeriatr. 2009;21:654-66.
13. Bliwise DL, Mercaldo ND, Avidan AY, Boeve BF, Greer SA, Kukull WA. Sleep disturbance in dementia with Lewy bodies and Alzheimer's disease: a multicenter analysis. Dement Geriatr Cogn Disord. 2011;31:239-46.

14. Moran M, Lynch CA, Walsh C, Coen R, Coakley D, Lawlor BA. Sleep disturbance in mild to moderate Alzheimer's disease. Sleep Med. 2005;6:347-52

15. Potvin $\mathrm{O}$, Lorrain $\mathrm{D}$, Forget $\mathrm{H}$, et al. Sleep quality and 1-year incident cognitive impairment in community-dwelling older adults. Sleep. 2012;35:491-9.

16. Saint MM, Sforza E, Barthelemy JC, Thomas-Anterion C, Roche F. Does subjective sleep affect cognitive function in healthy elderly subjects? The Proof cohort. Sleep Med. 2012;13:1146-52.

17. Malkki H. Alzheimer disease: sleep alleviates AD-related neuropathological processes. Nat Rev Neurol. 2013:9:657.

18. Neylan TC, Mueller SG, Wang Z, et al. Insomnia severity is associated with a decreased volume of the CA3/dentate gyrus hippocampal subfield. Biol Psychiatry. 2010;68:494-6.

19. Schliebs $R$, Arendt $T$. The significance of the cholinergic system in the brain during aging and in Alzheimer's disease. J Neural Transm. 2006;113:1625-44.

20. Moraes WS, Poyares DR, Guilleminault C, Ramos LR, Bertolucci PH, Tufik S. The effect of donepezil on sleep and REM sleep EEG in patients with Alzheimer disease: a double-blind placebo-controlled study. Sleep. 2006:29:199-205.

21. Cooke JR, Loredo JS, Liu L, et al. Acetylcholinesterase inhibitors and sleep architecture in patients with Alzheimer's disease. Drugs Aging. 2006:23:503-11.

22. Cardinali DP, Furio AM, Brusco LI. Clinical aspects of melatonin intervention in Alzheimer's disease progression. Curr Neuropharmacol. 2010;8:218.

23. Cardinali DP, Vigo DE, Olivar N, Vidal MF, Furio AM, Brusco L. Therapeutic application of melatonin in mild cognitive impairment. Am J Neurodegenerative Dis. 2012;1:280

24. Winblad B, Gauthier S, Scinto L, et al. Safety and efficacy of galantamine in subjects with mild cognitive impairment. Neurology. 2008;70:2024-35.

25. Peters $\mathrm{O}$, Lorenz $\mathrm{D}$, Fesche $\mathrm{A}$, et al. A combination of galantamine and memantine modifies cognitive function in subjects with amnestic $\mathrm{MCl}$. J Nutr Health Aging. 2012;16:544-8.

26. Honig LS, Vellas B, Woodward M, et al. Trial of solanezumab for mild dementia due to Alzheimer's disease. N Engl J Med. 2018;378:321-30.

27. He B, Xu F, Xiao F, et al. Neuroprotective effects of nootkatone from Alpiniae oxyphyllae Fructus against amyloid-beta-induced cognitive impairment. Metab Brain Dis. 2018:33:251-9.

28. Liu ZK, Ng CF, Shiu HT, et al. Neuroprotective effect of Da Chuanxiong Formula against cognitive and motor deficits in a rat controlled cortical impact model of traumatic brain injury. J Ethnopharmacol. 2018;217:11-22.

29. Guan D, Su Y, Li Y, et al. Tetramethylpyrazine inhibits $\mathrm{CoCl}_{2}$-induced neurotoxicity through enhancement of $\mathrm{Nrf2/GCLC/GSH}$ and suppression of HIF1alpha/NOX2/ROS pathways. J Neurochem. 2015;134:551-65.

30. Michel HE, Tadros MG, Esmat A, Khalifa AE, Abdel-Tawab AM. Tetramethylpyrazine ameliorates rotenone-induced Parkinson's disease in rats: involvement of its anti-inflammatory and anti-apoptotic actions. Mol Neurobiol. 2017;54:4866-78.

31. Ringman JM, Frautschy SA, Teng $E$, et al. Oral curcumin for Alzheimer's disease: tolerability and efficacy in a 24-week randomized, double blind, placebo-controlled study. Alzheimers Res Ther. 2012:4:43.

32. Vidal B, Vazquez-Roque RA, Gnecco D, et al. Curcuma treatment prevents cognitive deficit and alteration of neuronal morphology in the limbic system of aging rats. Synapse. 2017;71:e21952

33. Zhao C, Zhang H, Li H, et al. Geniposide ameliorates cognitive deficits by attenuating the cholinergic defect and amyloidosis in middle-aged Alzheimer model mice. Neuropharmacology. 2017;116:18-29.

34. Zang CX, Bao XQ, Li L, et al. The protective effects of Gardenia jasminoides (Fructus Gardenia) on amyloid-beta-induced mouse cognitive impairment and neurotoxicity. Am J Chin Med. 2018;46:389-405.

35. Zheng M, Qu L, Lou Y. Effects of icariin combined with Panax notoginseng saponins on ischemia reperfusion-induced cognitive impairments related with oxidative stress and CA1 of hippocampal neurons in rat. Phytother Res. 2008:22:597-604

36. Huang JL, Feng YQ, Bai LR, et al. Fraction n-butanol of Radix Notoginseng protects PC12 cells from Abeta $25-35$-induced cytotoxicity and alleviates cognitive deficits in SAMP8 mice by attenuating oxidative stress and Abeta accumulation. Evid Based Complement Alternat Med. 2017:2017:8469754.

37. Wu WB, Xie SL, Lei M, Zhang TM, Zhang WW. Research on extraction process of Yizhi Anshen oral liquid. Pharm Clin Chinese Materia Medica. 2016;7:27-30 (In simplified Chinese). 
38. Yang K, Xie SL, Zhang X, Lei M, Wu WB. Effects of Xiangshao capsules on hormone and 5-HT receptors in older female rats. Pharmacol Clin Chinese Materia Medica. 2015;31:207-10 (In simplified Chinese).

39. Frohlich EE, Farzi A, Mayerhofer $R$, et al. Cognitive impairment by antibioticinduced gut dysbiosis: analysis of gut microbiota-brain communication. Brain Behav Immun. 2016;56:140-55.

40. Cattaneo A, Cattane N, Galluzzi S, et al. Association of brain amyloidosis with pro-inflammatory gut bacterial taxa and peripheral inflammation markers in cognitively impaired elderly. Neurobiol Aging. 2017:49:60-8.

41. Mahmoudian Dehkordi S, Arnold M, Nho K, et al. Altered bile acid profile associates with cognitive impairment in Alzheimer's disease-an emerging role for gut microbiome. Alzheimers Dement. 2019;15:76-92.

42. Molnar FJ, Man-Son-Hing M, Fergusson D. Systematic review of measures of clinical significance employed in randomized controlled trials of drugs for dementia. J Am Geriatr Soc. 2009:57:536-46.

43. Miao YC, Tian JZ, Shi J, Mao M. Effects of Chinese medicine for tonifying the kidney and resolving phlegm and blood stasis in treating patients with amnestic mild cognitive impairment: a randomized, double-blind and parallel-controlled trial. Zhong Xi Yi Jie He Xue Bao. 2012;10:390-7.

\section{Publisher's Note}

Springer Nature remains neutral with regard to jurisdictional claims in published maps and institutional affiliations.

Ready to submit your research? Choose BMC and benefit from:

- fast, convenient online submission

- thorough peer review by experienced researchers in your field

- rapid publication on acceptance

- support for research data, including large and complex data types

- gold Open Access which fosters wider collaboration and increased citations

- maximum visibility for your research: over $100 \mathrm{M}$ website views per year

At BMC, research is always in progress.

Learn more biomedcentral.com/submissions 